\title{
The rare case of a large complex intraosseous cranial arteriovenous malformation with successful multidisciplinary management
}

\author{
Anas Al-Smadi, MD, ${ }^{1}$ Tahaamin Shokuhfar, MD, ${ }^{1}$ Andrew Johnston, BS, ${ }^{1}$ Tord D. Alden, MD, ${ }^{2}$ \\ Robin Bowman, MD, ${ }^{2}$ and Ali Shaibani, MD ${ }^{1,2}$ \\ Departments of ${ }^{1}$ Radiology and ${ }^{2}$ Neurological Surgery, Northwestern University, Feinberg School of Medicine, Chicago, Illinois \\ Intraosseous cranial arteriovenous malformations (AVMs) are very rare, challenging entities. The authors report the case \\ of an extracranial parietooccipital vascular lesion. A 12-year-old boy presented with accelerated growth of a right scalp \\ lesion over a few months. Digital subtraction angiography showed a large, right parietooccipital intraosseous AVM with \\ multiple complex arterial feeders. Treatment of these lesions is difficult and can necessitate a multidisciplinary approach. \\ In the featured case, 6 embolization procedures were performed over 1 year, including both transarterial and transve- \\ nous approaches, followed by total resection. The authors describe what is thought to be the second case of an AVM \\ originating in the cranial bones and the first case with successful multidisciplinary management. \\ https://thejns.org/doi/abs/10.3171/2017.7.PEDS17161
}

KEY WORDS arteriovenous malformation; angiography; embolization; intraosseous; neurological surgery; vascular disorders

$\Lambda$ RTERIOVENOUS malformations (AVMs) are fast-flow vascular malformations composed of a complex vascular network that connects feeding arteries directly to draining veins. The normal intervening capillary network is absent. They most commonly affect the head and neck (47.4\%), ${ }^{14}$ whereas extracranial AVMs account for about $4.7 \%$ of all AVMs, according to one study..$^{15}$ Primary intraosseous AVMs are rare, accounting for less than $1 \%$ of all primary intraosseous vascular lesions ${ }^{13}$ but can cause various complications such as hemorrhage, bony alterations, and congestive heart failure. ${ }^{3,713}$ The most common reported location for these intraosseous vascular malformations is the facial bones; however, intraosseous AVMs have been reported in other skeletal sites, including the humerus, radius, femur, tibia, and spine. ${ }^{7,16}$ The management of extradural intraosseous AVMs of the head and neck is challenging and can necessitate a multidisciplinary approach. Combined embolization and resection represents the "first-line" therapy for many AVMs. ${ }^{1}$ In this report, we describe the case of a large and complex extradural, parietooccipital intraosseous cranial AVM that was treated using a combination of embolization and surgery.

\section{Case Report}

History and Examination

A 12-year-old boy presented with a right parietooccipital mass that was first noticed 2 years earlier as a small, hard bump on the right side of his head. The bump was routinely measured by his pediatrician and found to be stable. The mass began to increase in size 3 months before presentation to us, with a new posterior auricular mass developing several weeks before assessment. The patient's mother also noticed a bluish discoloration of his ipsilateral ear a few days prior to the initial visit. The patient's medical history was positive only for the diagnosis of attention-deficit/hyperactivity disorder (ADHD) managed with medication, and there was no family history of AVM or other vascular malformations. Physical examination was remarkable for a large right-sided, subcutaneous, soft pulsatile mass with a thrill that extended from the midparietal bone down toward the mastoid and then medially extending as far as the inion. It was a nontender mass with bluish discoloration of the focal swelling superficial to the mastoid. The patient was otherwise asymptomatic

ABBREVIATIONS $A V M=$ arteriovenous malformation; $E C A=$ external carotid artery; $M M A=$ middle meningeal artery; $N B C A=N$-butyl cyanoacrylate; OccA $=0 c c i p i t a l$ artery; STA = superficial temporal artery.

SUBMITTED March 28, 2017. ACCEPTED July 7, 2017

INCLUDE WHEN CITING Published online September 29, 2017; DOI: 10.3171/2017.7.PEDS17161. 
and showed no neurological symptoms. Head CT obtained by his pediatrician revealed a right parietooccipital extracranial vascular lesion and a widening overgrowth of the subjacent skull (Fig. 1 left).

\section{Angiography}

The patient was referred to Interventional Neuroradiology for further workup. Diagnostic angiography, which included 3D and DynaCT (Siemens), was performed (Fig. 2). Analysis of the images revealed a large AVM $(8 \times 10$ $\mathrm{cm}$ ) with the nidus centered within the calvaria, involving the right parietal bone. As is often seen with hyperemic lesions within or adjacent to bone, there was consequent growth and increased thickness of the calvaria. As demonstrated in Fig. 2, direct supply to the AVM was noted from the right occipital artery (OccA), the right superficial temporal artery (STA), both middle meningeal arteries (MMAs), a meningeal branch of the right anterior deep temporal artery, and the right-sided posterior falcine and meningeal arteries (arising from the posterior inferior cerebellar artery as an anatomical variant). Indirect supply was seen from the left OccA and left STA. Venous drainage was initially into osseous veins, which then converged into a dilated tortuous network of scalp veins. The most dominant scalp vein extended from the right parietal region inferiorly to the right mastoid region with an attenuated connection to a branch of the right external jugular vein. Additional scalp veins were noted along the posterior aspect of the scalp, the right temporal region, the left side of the scalp extending to the forehead, the left angular vein, and retrograde into the superior ophthalmic vein and cavernous sinus with antegrade drainage down the left inferior petrosal sinus. The right OccA showed at least 9 flow-related aneurysms along its course. A small flowrelated aneurysm was also associated with the right STA.

\section{Embolization}

Embolization of scalp vessels (STA, OccA) with liquid embolics is challenging given the possibility of soft tissue and skin necrosis. While some embolization of the lesion was performed in the scalp arteries, much of the nidal filling was achieved using the meningeal arteries. The large size of the AVM required a total of 6 endovascular embolization procedures over 1 year (Figs. 3 and 4), resulting in a $70 \%-80 \%$ reduction in size of the patent nidus, followed by resection. Figure 1 right shows an MRI study that was done after the fifth embolization procedure and reveals the extent of the intraosseous AVM nidus. The last embolization procedure was performed on the day prior to surgery. A total of 10 arterial pedicles were embolized. Two transvenous embolizations with retrograde injection of an embolic agent were performed. Onyx (Medtronic) and $\mathrm{N}$-butyl cyanoacrylate (NBCA) were used for nidal penetration. Polyvinyl alcohol particles were used in the final preoperative embolization in scalp vessels providing residual collateral supply to the AVM. No procedure caused significant complications, though the patient experienced transient double vision following the fifth procedure. An episode of headache and emesis followed the sixth procedure but was resolved with antiemetics. Neurological examination remained normal.
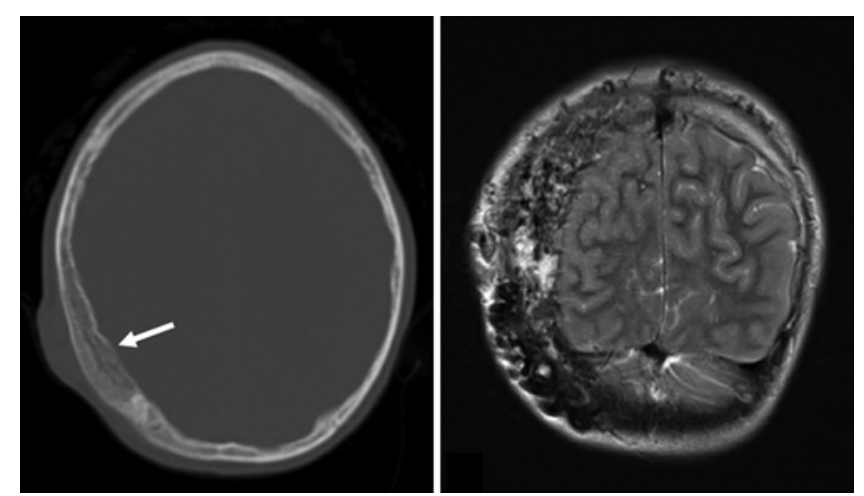

FIG. 1. Left: Preoperative bone window CT shows overgrowth of the occipitotemporal bone due to the AVM nidus within (arrow). Right: Coronal T2-weighted MR image obtained after the fifth embolization procedure, showing the extent of the nidus.

Given the multiple embolization procedures, there was concern about diminished blood supply to the right parietal scalp. Preoperative physical assessment of scalp vascularization revealed no evidence of poor capillary refill.

\section{Resection}

The day after the final embolization, a right-sided craniectomy and resection of the extradural AVM was performed using titanium mesh and methylmethacrylate cranioplasty. The neurosurgery team took extra precautions to maintain coverage of the defect with scalp so that no incision was made over an area that was reconstructed. They tried to maintain any feeder from the OccA and STA. A very thick skull filled with embolization material was noted at surgery. It was soft and easily curetted, and the neurosurgeon went around the perimeter to minimize bleeding. Intraoperative examination revealed no evidence of any AVM dural extension, and the whole nidus was contained inside the bone. Postoperative diagnostic angiography showed no evidence of residual lesions (Fig. 5). Figure 6 shows the large bony craniectomy defect repaired using titanium mesh. The patient was successfully followed up by the plastic and neurosurgery teams and showed complete wound healing, and the bony construction looked good cosmetically. Some scalp veins that had been embolized were still present, but the patient was not interested in removing them.

\section{Discussion}

Arteriovenous malformations are dynamic vascular anomalies that tend to progress before adulthood, especially during adolescence; Liu and colleagues report that the average age at which AVMs progress is 12.7 years. ${ }^{9}$ The primary area of skeletal involvement of extradural AVM is teeth-bearing bones. ${ }^{7}$ We found only one other case report in the English literature describing an AVM centered in the cranial bones. ${ }^{3}$

Although AVMs show stability and normal endothelial activity on the cellular level, they can be devastating from a clinical standpoint, particularly when there is high-volume AV shunting. Complications of extradural AVMs include 

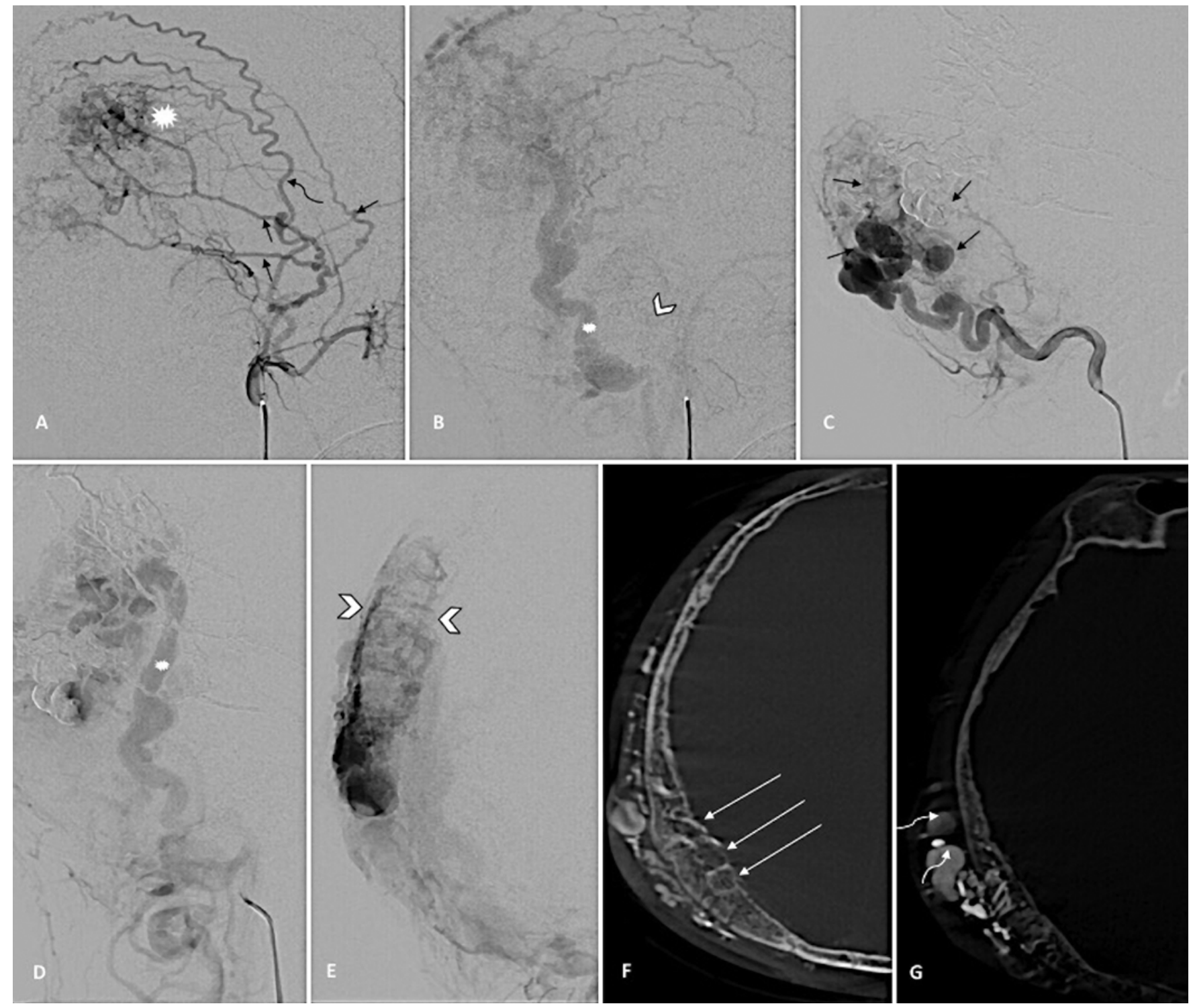

FIG. 2. Angiography studies. A: Arterial and venous phases of AVM flow, from a right external carotid artery (ECA) injection distal to the occipital artery (OccA), demonstrating direct supply from the right middle meningeal artery (MMA; straight arrows) and the superficial temporal artery (STA; curved arrow), as well as a flow-related aneurysm associated with the STA. White star indicates part of the AVM nidus. B: The dominant scalp draining vein extends to connect with a branch of the right ECA via a stenotic connection (arrowhead). The dilated portion of the vein just proximal to the connection was visible as a bluish bump behind the external ear (white star). C: Right OccA angiogram, arterial and venous phases of AVM flow, demonstrating multiple flow-related aneurysms along the course of the right OccA (small arrows). D: Multiple scalp draining veins, most of which converge on the dominant draining vein (white star). E: Right OccA angiogram, oblique view, demonstrating the nidus within the bone. Inner and outer tables are marked by arrowheads. F: DynaCT axial reconstructions with injection of 30\% contrast in the right ECA. Multiple nidal channels appear within the skull (long arrows). G: Draining veins in the scalp (curved arrows). There is bony overgrowth and thickening of the involved portions of the skull.

pain, hemorrhage, disfigurement, ulceration, alteration of tissues, obstruction of vital structures, and, in rare cases, cardiac overload. ${ }^{9}$ Schobinger classified AVMs clinically into 4 stages: Stage I is a stable AVM (quiescence), Stage II begins when the AVM enlarges and/or becomes pulsatile (expansion), Stage III occurs when painful or dystrophic skin changes like ulceration or bleeding develop (destruction), and Stage IV is characterized by heart failure (decompensation). ${ }^{9}$ Advancement in the clinical presentation of an AVM into a higher Schobinger stage is considered "progression." The patient in our case progressed from Stage I to Stage II given the pulsatile and expanding nature of the lesion.

Expansion of AVMs is the main cause of morbidity. They can enlarge due to a variety of factors, including increased blood flow that leads to collateralization, thickening and dilation of adjacent vessels, and neovascularization. The increase of circulating hormones during puberty can contrib- 

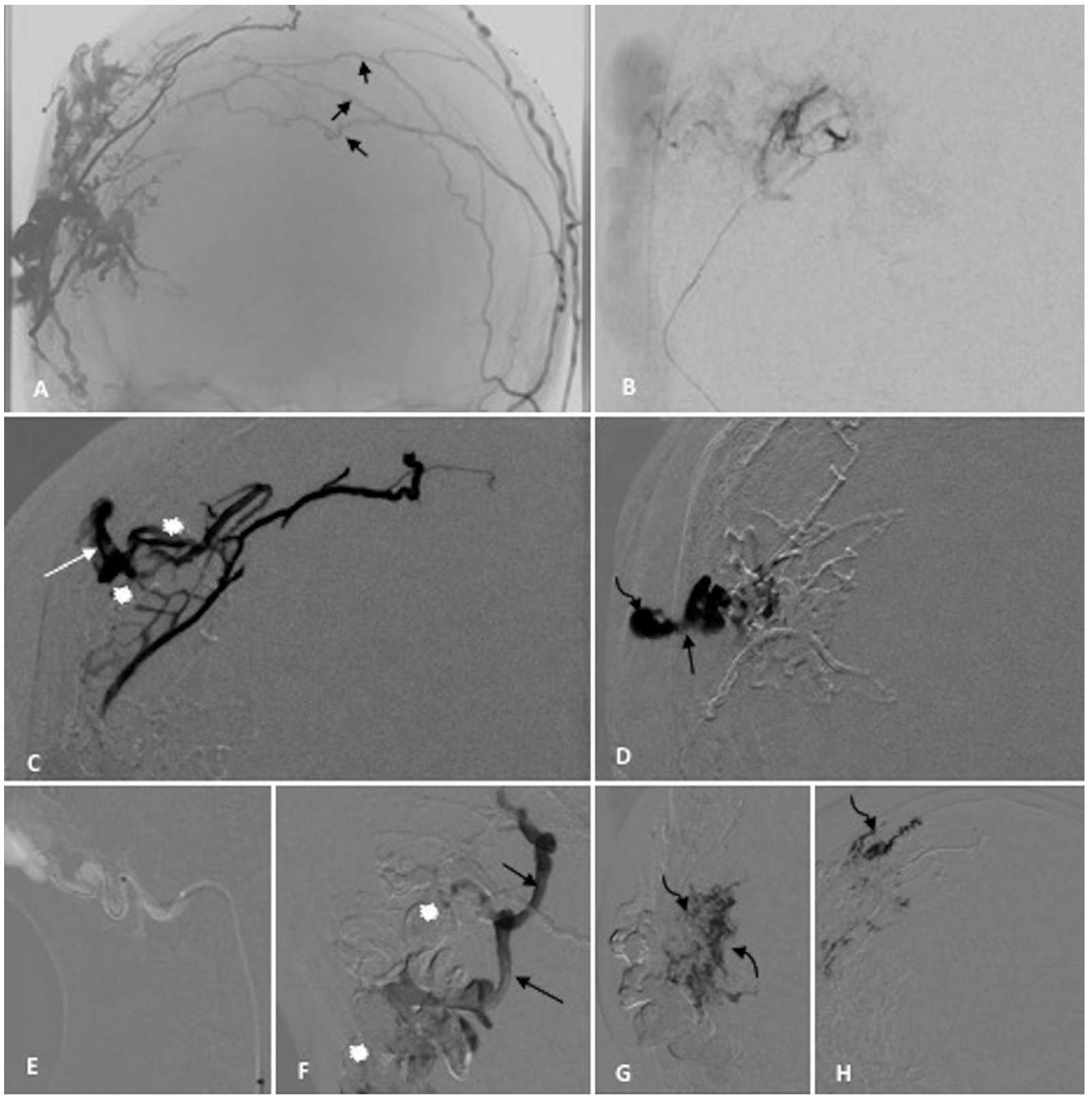

FIG. 3. Embolization. Anteroposterior unsubtracted view, left ECA injection (A), demonstrating enlarged branches of the left MMA connecting across the midline (arrows) with distal branches of the right MMA, supplying the AVM. Onyx appears within portions of the AVM nidus and some draining veins. Microcatheter angiogram (B) of a distal branch of the right MMA, demonstrating supply to the AVM nidus in the skull, with venous drainage into scalp veins. Roadmap images (C-D) obtained during injection of Onyx via an MMA approach, demonstrating filling of the nidus (white stars) and extension of Onyx transosseously (arrow) into a scalp draining vein (curved arrow). Transarterial embolization (E-H) from a right OccA approach, using a Scepter balloon-tipped microcatheter (MicroVention). Roadmap image (E) showing position of the microcatheter before inflation of the balloon. The Onyx cast is filling multiple flow-related aneurysms ( $\mathrm{F}$, white stars), extending into distal arterial branches ( $\mathrm{F}$, straight arrows) and the intraosseous nidus (G-H, curved arrows).

ute to AVM enlargement, possibly by enhancing neovascularization by promoting vascular endothelial growth factor (VEGF) production and leading to endothelial proliferation. ${ }^{5,6,9,10}$ A retrospective study showed a 2 -fold increased risk of progression in both males and females during puberty. ${ }^{9}$ The role of circulating hormones could explain the rapid progression in AVM size in our patient.
Another known stimulator of angiogenesis is ischemia, which can cause enlargement of AVMs after arterial ligation or trauma. $7,11,14$ The senior author noticed neovascularization and an increased nidus size after the third embolization procedure, which could be explained by the stimulant effect of ischemia after embolizing feeding arteries. 


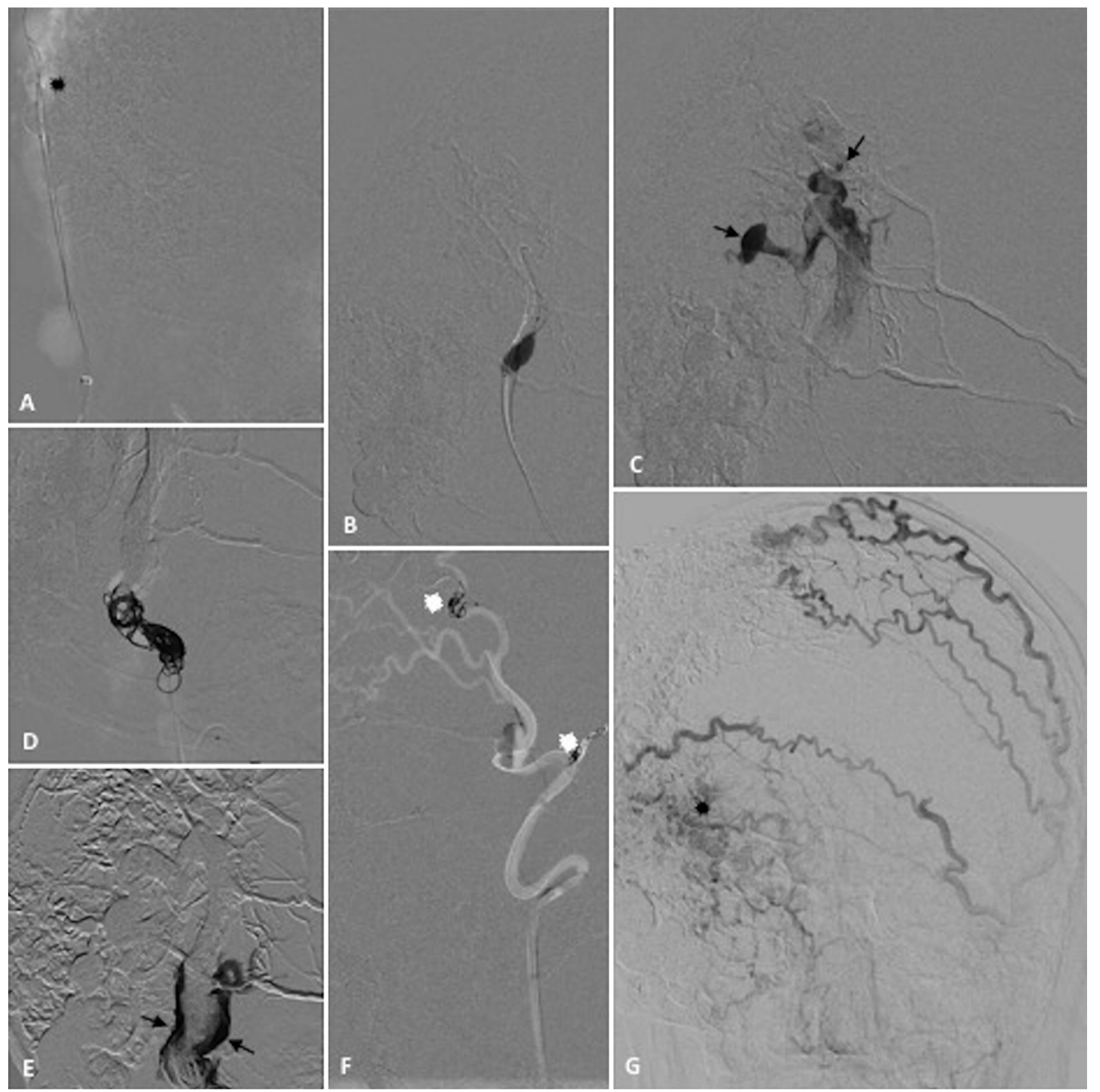

FIG. 4. Transvenous embolization of the AVM through the dominant scalp draining vein was performed twice. Roadmap image (A) demonstrating two 7-mm Scepter balloon-tipped microcatheters placed proximally in the main draining vein (black star). Roadmap image (B) obtained after inflation of both balloons, which was done to achieve relative flow obstruction downstream and push the embolic agent upstream toward the nidus. Onyx cast partway during transvenous retrograde embolization (C), extending into more proximal draining veins (arrows). Second session of the transvenous embolization (D-E), more distally in the dominant draining veins. Coils have been delivered distal to the microcatheter to achieve some downstream flow obstruction and facilitate retrograde injection of the embolic agent (arrows). Final preoperative particle embolization of scalp collateral supply; roadmap image (F) showing proximal coil occlusion of right STA branches not involved in AVM supply, to protect the scalp and subcutaneous soft tissues (white stars). Left OccA angiogram (G), anteroposterior view, obtained during particle embolization of collateral scalp supply to the AVM as the final preoperative embolization, demonstrating the enlarged OccA branches extending across the midline to supply residual nidus (black star).

The successful management of extradural AVMs may require an interdisciplinary approach. Of the few reported cases of intraosseous AVMs, most were treated using a combination of embolization and resection, ${ }^{8,13}$ although the case of an AVM in the petrous part of the temporal bone managed by embolization alone was followed by fa- cial paralysis and hoarseness. ${ }^{3}$ Embolization is not without risk, and potential complications include embolic obstruction of nontarget arteries, inflammation of local and perivascular tissues, and soft-tissue tattooing. ${ }^{2}$ Complete resection after embolization, when feasible, has thus become the most accepted treatment. ${ }^{14}$ Diffuse AVMs, as seen in 


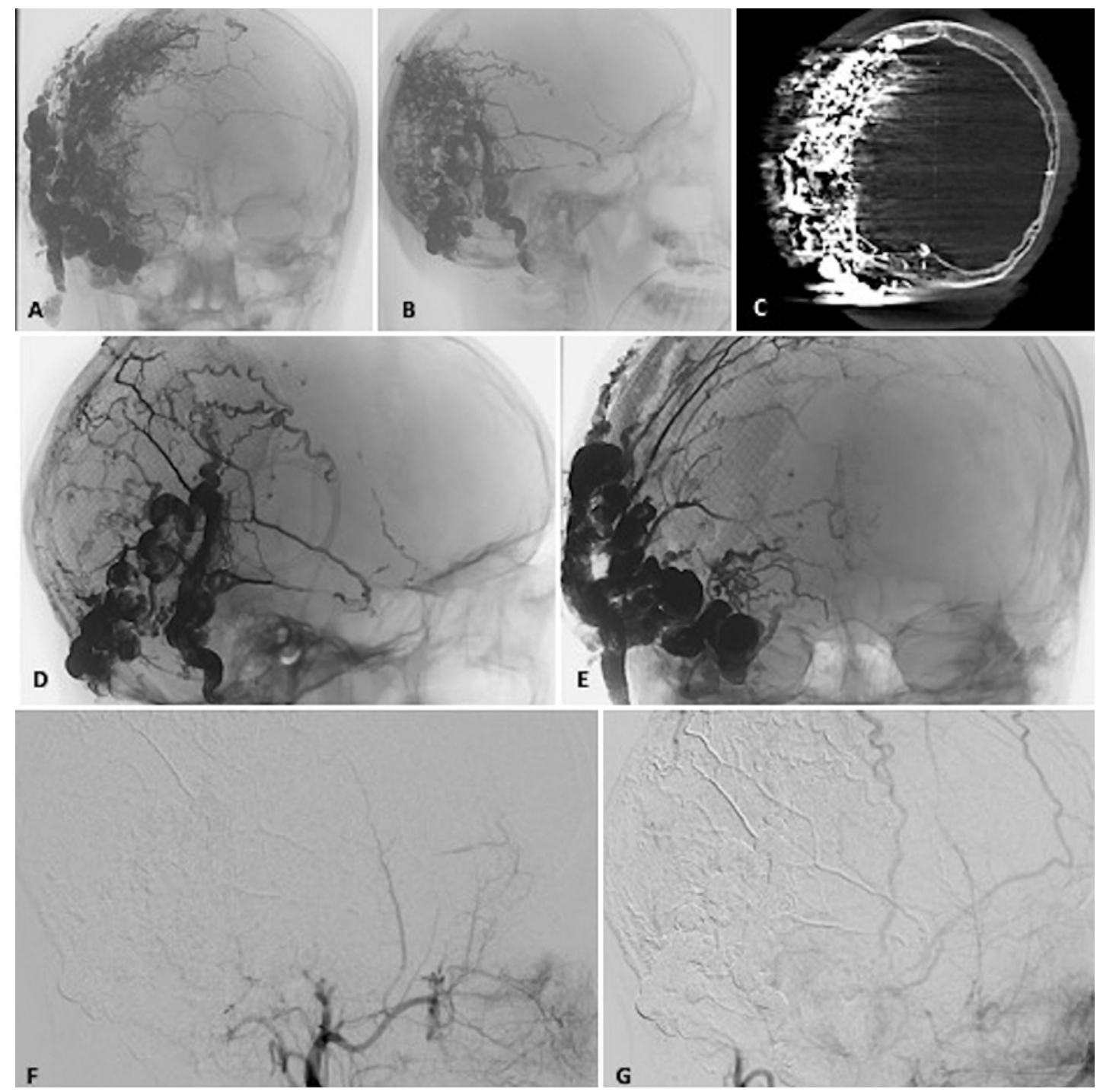

FIG. 5. Final embolization and postoperative angiography. Unsubtracted anteroposterior and lateral views (A-B) demonstrating the final cast of Onyx and NBCA. DynaCT coronal reconstruction $(\mathbf{C})$ obtained after final embolization, demonstrating the highattenuation embolic agent (primarily Onyx) spanning the inner table within meningeal branches, through the skull and outer table, into the scalp veins. Unsubtracted lateral and anteroposterior views (D-E) obtained after resection of the nidus, with mesh and polymethylmethacrylate reconstruction of the skull defect. Residual embolic material appears in the arteries and veins proximal and distal to the resected nidus. Digital subtraction angiogram, lateral views of right $(\mathbf{F})$ and left $(\mathbf{G}) \mathrm{ECA}$, obtained postresection, showing no evidence of residual AV shunting.

our patient, with their multiple arterial feeders and diffuse skin involvement, ${ }^{14}$ are much more challenging to treat, have higher recurrence rates after treatment, and are associated with a higher risk of surgical morbidity. ${ }^{12}$ Therefore, they can require more extensive embolization procedures to control.

Embolization aids in the achievement of total resection and decreases the risk of recurrence; additionally, it significantly reduces the risk of intraoperative bleeding. ${ }^{4,14}$ Total resection of the nidus is critical, considering that Goldenberg et al. reported a regrowth rate of $18.2 \%$ in total resection cases compared with a rate of $66.7 \%$ when partial resection was performed. ${ }^{4,7}$ Given the potential side effects of treatment, interventional management for AVMs is often reserved for progressive lesions of Schobinger Stage II and above. ${ }^{1}$ Resection within 24-48 hours of embolization is ideal for delineating lesions and preventing acute inflammation caused by embolic materials..$^{14}$ The final embolization procedure was performed within 24 hours of complete AVM resection.

\section{Conclusions}

In summary, intraosseous AVMs are rare events. To the best of our knowledge, we report the second case of an AVM originating in the cranial bones. The ideal manage- 

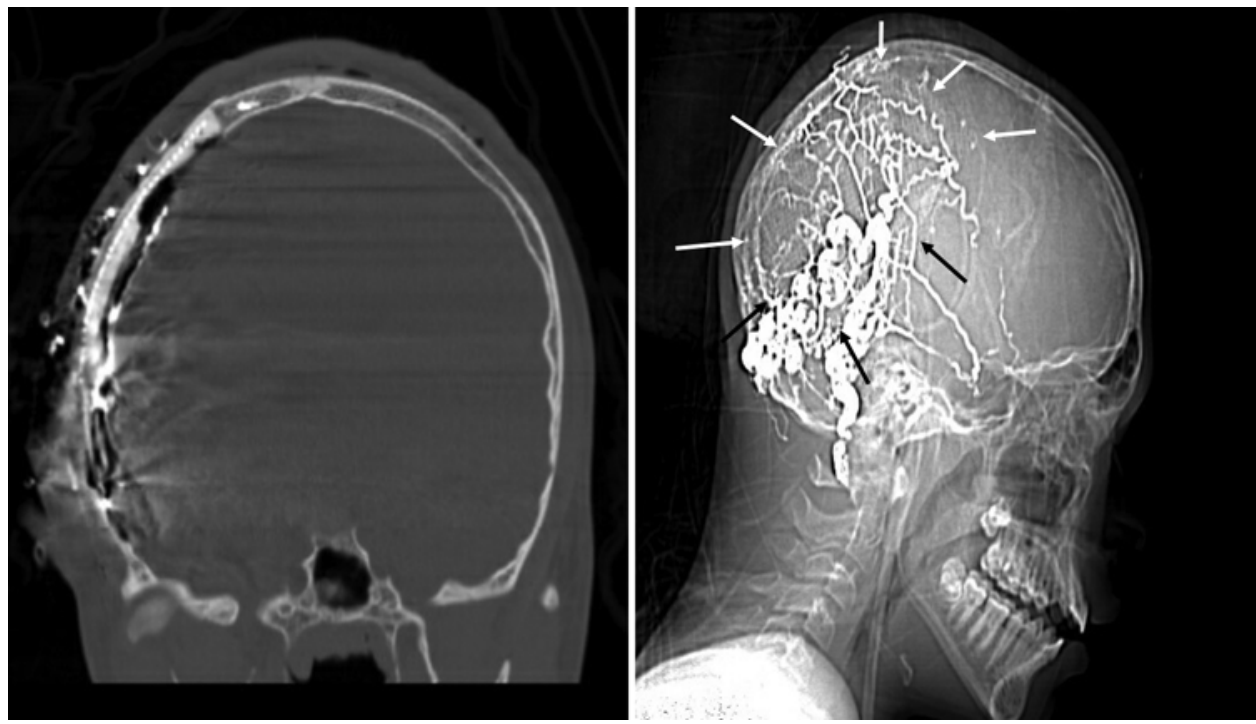

FIG. 6. Mesh and polymethylmethacrylate were used to repair the large bony craniectomy defect (outlined by arrows) after resection of the intraosseous AVM.

ment of these lesions may be embolization followed by complete resection when feasible.

\section{References}

1. Fowell C, Jones R, Nishikawa H, Monaghan A: Arteriovenous malformations of the head and neck: current concepts in management. Br J Oral Maxillofac Surg 54:482-487, 2016

2. Fowell C, Jones R, Willis A, Monaghan A: Arteriovenous malformations of the head and neck: experiences in modern management and a review of current concepts and novel treatment modalities. Int J Oral Maxillofac Surg 44 (Suppl 1):e61-e62, 2015

3. Gokce EC, Cemil B, Kirbas I, Bozkurt A, Erdogan B: A rare case of intracerebral hemorrhage due to arteriovenous malformation located at petrous portion of the temporal bone. Turk Neurosurg 23:811-814, 2013

4. Goldenberg DC, Hiraki PY, Caldas JG, Puglia P, Marques TM, Gemperli R: Surgical treatment of extracranial arteriovenous malformations after multiple embolizations: outcomes in a series of 31 patients. Plast Reconstr Surg 135:543-552, 2015

5. Hashimoto T, Wu Y, Lawton MT, Yang GY, Barbaro NM, Young WL: Coexpression of angiogenic factors in brain arteriovenous malformations. Neurosurgery 56:1058-1065, 2005

6. Hyder SM, Nawaz Z, Chiappetta C, Stancel GM: Identification of functional estrogen response elements in the gene coding for the potent angiogenic factor vascular endothelial growth factor. Cancer Res 60:3183-3190, 2000

7. Kohout MP, Hansen M, Pribaz JJ, Mulliken JB: Arteriovenous malformations of the head and neck: natural history and management. Plast Reconstr Surg 102:643-654, 1998

8. Lemound J, Brachvogel P, Götz F, Rücker M, Gellrich NC, Eckardt A: Treatment of mandibular high-flow vascular malformations: report of 2 cases. J Oral Maxillofac Surg 69:1956-1966, 2011

9. Liu AS, Mulliken JB, Zurakowski D, Fishman SJ, Greene AK: Extracranial arteriovenous malformations: natural progression and recurrence after treatment. Plast Reconstr Surg 125:1185-1194, 2010

10. Mueller MD, Vigne JL, Minchenko A, Lebovic DI, Leitman DC, Taylor RN: Regulation of vascular endothelial growth factor (VEGF) gene transcription by estrogen receptors $\alpha$ and $\beta$. Proc Natl Acad Sci U S A 97:10972-10977, 2000

11. Mulliken JB, Glowacki J: Hemangiomas and vascular malformations in infants and children: a classification based on endothelial characteristics. Plast Reconstr Surg 69:412-422, 1982

12. Richter GT, Suen JY: Pediatric extracranial arteriovenous malformations. Curr Opin Otolaryngol Head Neck Surg 19:455-461, 2011

13. Savader SJ, Savader BL, Otero RR: Intraosseous arteriovenous malformations mimicking malignant disease. Acta Radiol 29:109-114, 1988

14. Uller W, Alomari AI, Richter GT: Arteriovenous malformations. Semin Pediatr Surg 23:203-207, 2014

15. Visser A, FitzJohn T, Tan ST: Surgical management of arteriovenous malformation. J Plast Reconstr Aesthet Surg 64:283-291, 2011

16. Wang HH, Yeh TT, Lin YC, Huang GS: Imaging features of an intraosseous arteriovenous malformation in the tibia. Singapore Med J 56:e21-e25, 2015

\section{Disclosures}

The authors report no conflict of interest concerning the materials or methods used in this study or the findings specified in this paper.

\section{Author Contributions}

Conception and design: Shaibani, Al-Smadi. Acquisition of data: Shaibani, Al-Smadi, Alden, Bowman. Analysis and interpretation of data: Shaibani, Al-Smadi, Shokuhfar, Johnston, Bowman. Drafting the article: Al-Smadi, Shokuhfar, Johnston. Critically revising the article: Shaibani, Al-Smadi, Shokuhfar, Johnston, Alden. Reviewed submitted version of manuscript: Shaibani, AlSmadi, Shokuhfar, Johnston, Alden. Approved the final version of the manuscript on behalf of all authors: Shaibani. Administrative/ technical/material support: Alden, Bowman. Study supervision: Shaibani, Bowman.

\section{Correspondence}

Ali Shaibani, Northwestern University, Feinberg School of Medicine, 676 N St. Clair St., Ste. 1400, Chicago, IL 60611. email: ashaiban@nm.org. 